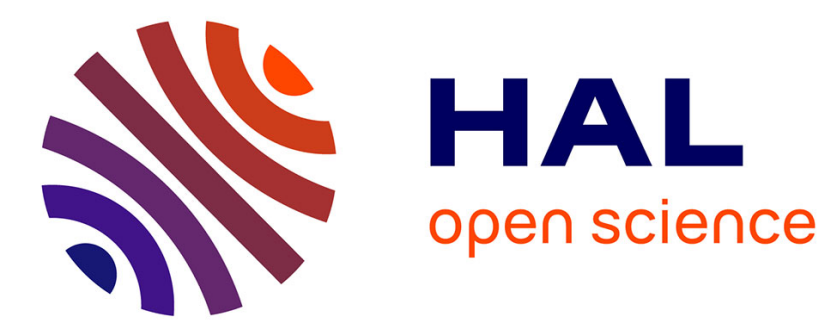

\title{
Optical signatures of borico dyes: a TD-DFT analysis
}

\author{
Azzam Charaf-Eddin, Boris Le Guennic, Denis Jacquemin
}

\section{To cite this version:}

Azzam Charaf-Eddin, Boris Le Guennic, Denis Jacquemin. Optical signatures of borico dyes: a TDDFT analysis. Theoretical Chemistry Accounts: Theory, Computation, and Modeling, 2014, 133, pp.1456. 10.1007/s00214-014-1456-y . hal-00998543

\section{HAL Id: hal-00998543 https://hal.science/hal-00998543}

Submitted on 2 Jun 2014

HAL is a multi-disciplinary open access archive for the deposit and dissemination of scientific research documents, whether they are published or not. The documents may come from teaching and research institutions in France or abroad, or from public or private research centers.
L'archive ouverte pluridisciplinaire HAL, est destinée au dépôt et à la diffusion de documents scientifiques de niveau recherche, publiés ou non, émanant des établissements d'enseignement et de recherche français ou étrangers, des laboratoires publics ou privés. 


\title{
Optical signatures of borico dyes: a TD-DFT analysis
}

\author{
Azzam CHARAF-EDDIN · Boris LE GUENNIC · Denis JACQUEMIN
}

Received: date / Accepted: date

\begin{abstract}
Using Time-Dependent Density Functional Theory (TD-DFT), we investigate the excited-state properties of a series of emissive dyes combining the properties of coumarins and fluoroborate compounds. These boron-iminocoumarins (borico) compounds have been synthesized very recently by Frath and coworkers [Chem. Commun., 49 (2013) 4908]. We determine both their vertical and 0-0 energies, reproduce and analyze their characteristic experimental band shape, investigate the nature of the excited-states in large dyads containing two different fluoroborate complexes and design redshifted compounds. We also consider an additional panel of fused coumarin-BODIPY emitters.
\end{abstract}

Keywords BORICO · Dyes · Vibronic effects · Time-Dependent Density Functional Theory

\section{Introduction}

Despite its venerable age, dye chemistry remains a field of intense research with a specific focus set on fluorophores during the last decades. In the category of

A. Charaf-Eddin · D. Jacquemin

Laboratoire CEISAM - UMR CNRS 6230, Université de Nantes, 2 Rue de la Houssinière, BP 92208, 44322 Nantes Cedex 3, France

B. Le Guennic

Institut des Sciences Chimiques de Rennes, UMR 6226 CNRS-Université de Rennes 1, 263 Av. du General Leclerc, 35042 Rennes Cedex, France

D. Jacquemin

Institut Universitaire de France, 103, bd Saint-Michel, F75005 Paris Cedex 05, France.

Tel.: +33-2-51-12-55-64

E-mail: Denis.Jacquemin@univ-nantes.fr strongly emissive compounds, fluoroborate complexes that present a $\mathrm{BF}_{2}$ group tethered between two electronegative moieties, typically nitrogen or/and oxygen atoms, have been actively investigated. Indeed, BODIPY (boron-dipyrromethene), aza-BODIPY and boranil derivatives, that can all be viewed as constrained cyanines, have become key tools for biological, medicinal and analytical applications due to their excellent photophysical properties $[1,2]$. These molecules that are highly photostable and develop very large quantum yields, have found applications in several fields, e.g., ion sensing $[3,4]$, nonlinear optics [5], photodynamic therapy [6], as well as fluorescence imaging [7]. Of course, more traditional series of fluorescent dyes have already found practical applications, and the two most well-known families of organic fluorophores remain coumarins and naphthalimides, that can be used as paper brighteners $[8,9]$. In 2013, Frath and coworkers have proposed an original series of dyes combining (imino)coumarins to fluoroborate complexes, and named this new family borico [10]. These dyes, sketched in Figure 1, display both large absorption coefficients and fluorescence quantum yields going up to 0.81 . Frath et al. have also designed two dyads, one with a boranil moiety $(\mathbf{3})$ and one with a BODIPY (4). To the best of our knowledge, there is absolutely no previous theoretical investigation of these exciting structures, and this contribution aims to fill this gap. Note that, we found two other works dealing with joint coumarin-BODIPY emitters: on the one hand, the 2011 anion-sensing dimer of Cao et al. in which the two chromogens are separated by a short conjugated segment [11] and, on the other hand, the very recent work of Bochkov and coworkers who synthesized four different fused coumarin-BODIPY dyes [12], that we model in the last Section of the present paper. 


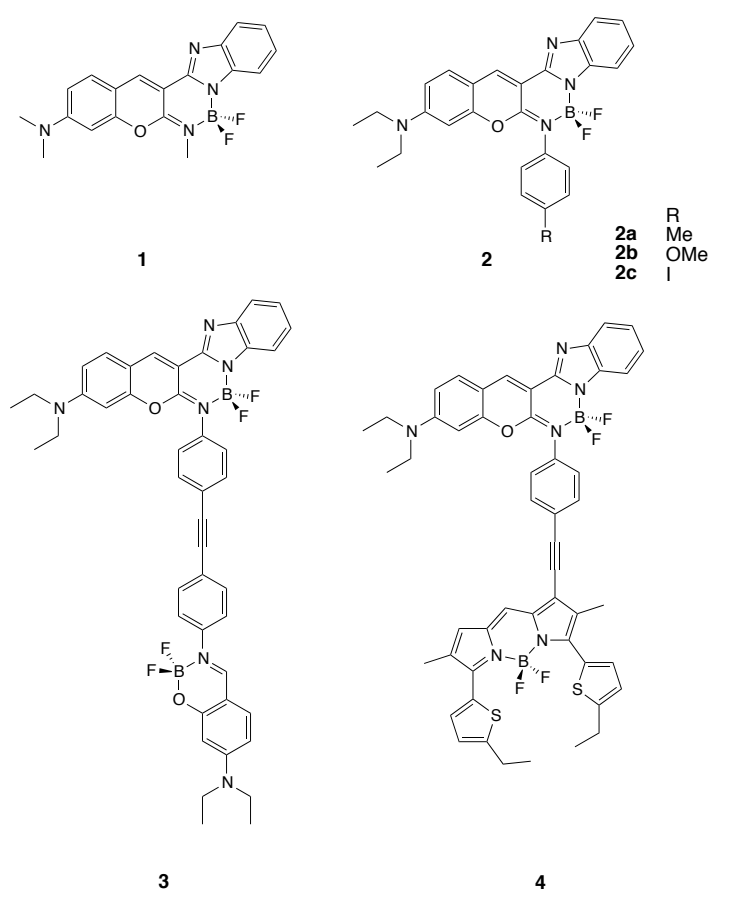

Fig. 1 Representation of the systems investigated herein. Molecules 2-4 have been synthesized [10], whereas $\mathbf{1}$ is a model compound presenting the borico core.

Amongst all possible first-principle approaches for investigating excited-state properties, Time-Dependent Density Functional Theory (TD-DFT) is certainly the most popular, with a continuously increasing number of published applications [13-16]. Beyond its widespread access in several quantum-chemical programs, three factors might explain the success of TD-DFT: 1) the possibility to tackle very extended molecules, e.g., possessing 300 atoms or even more if simplified approaches are used $[17,18] ; 2)$ the ease to combine TD-DFT with several environmental models allowing to accurately account for solvent and/or biochemical media [19,20]; 3) the availability of analytic first and second derivatives, allowing the optimization of the excited-state structures, as well as the determination of their vibrational patterns at a relatively small computational cost [2125]. No other excited-state approach, but the much less accurate Configuration Interaction Singles (CIS) scheme, combines these key advantages. Of course, TDDFT has its limitations, e.g., the difficulty to tackle cyanine excited-states, transitions involving double excitations, or, of course, multi-reference systems [26-28]. These limitations are related, on the one hand, to the use of the adiabatic approximation in most TD-DFT applications (that is the application of Casida's linearresponse approximation to TD-DFT), [13] and, on the other hand, to the selection of a specific approximate exchange-correlation functional. Nevertheless, for most valence transitions, TD-DFT provides accurate values with typical errors in the $0.1-0.3 \mathrm{eV}$ range. With TD$\mathrm{DFT}$, it is possible to determine both band shapes, through Franck-Condon calculations [29-31], and 0-0 energies, that are the difference between vibrationallycorrected excited- and ground-state energies at their respective optimal geometries [32-36]. These $0-0$ energies can be straightforwardly compared to the experimental crossing point between absorption and fluorescence curves (AFCP), if an appropriate methodology is used [33-36]. Of course, such computation protocol, that we used here, remains less applied than "straightforward" vertical TD-DFT calculations that do not require calculations of the zero-point vibrational energies (ZPVE) but only yield qualitative trends [16].

\section{Methodology}

All computations have been performed with the Gaussian 09 program package [37], applying default thresholds except for a tighten self-consistent field convergence $\left(10^{-9}\right.$ a.u. $)$, an improved optimization threshold $\left(10^{-5}\right.$ a.u. on average forces) and a more refined numerical DFT integration grid [so-called ultrafine grid, a pruned $(99,590)$ grid]. We have performed benchmarks previously for determining the most suited exchangecorrelation functional for fluoroborate emissive dyes [38, 39]. It turned out that Zhao and Truhlar's M06-2X meta-GGA hybrid [40] is clearly one of the most adequate and it was applied throughout in the following. Let us nevertheless underline, that for 0-0 energies, M06-2X tend to slightly overestimate the experimental data, with a typical mean absolute deviation of ca. $0.25 \mathrm{eV}$, though it yields very consistent results (large correlation). [35] Our computational protocol used to obtain the 0-0 energies by simultaneously accounting for vibrational and non-equilibrium solvation effects at the corrected linear-response (cLR) level [41], has been described elsewhere [35], and it relies on the use of two atomic basis sets: a first to determine the geometrical and vibrational parameters and a larger basis set to compute vertical transition energies. Previously [42], we have selected the $6-31 \mathrm{G}(\mathrm{d})$ and $6-311+\mathrm{G}(2 \mathrm{~d}, \mathrm{p})$ atomic basis sets as small and large basis sets, respectively, and this choice has been tested again here (see next Section). Note that for the iodine atom of 2c, the triple- $\zeta$ compact effective potential (CEP-121G) pseudopotential has been applied $[43,44]$. Solvent effects (dichloromethane consistently with experiment) have been accounted for with the well-known Polarizable Continuum Model (PCM) model [19]. In PCM-TD-DFT calculations, one can apply two limits, namely, the equilibrium (eq) and non-equilibrium (neq) approaches. In 
the latter, only the electrons of the solvent do adapt to the new electronic configuration of the solute (fast process). Clearly, the vertical transition energies better correspond to a neq scheme. On the contrary, in the eq approach, the solvent has time to adapt to the new electronic configuration of the solute (slow process). This latter limit is recommended for calculating excited-states geometries, vibrational signatures and consequently the 0-0 energies. In addition, we use, on top of the standard LR-PCM approach, the cLR-PCM to compute excited-state energies [41]. Again, the interested reader will find more details in Ref. [35].

The density difference plots shown below have been obtained by computing the difference between the excitedstate and ground-state total electronic densities. Note that the excited-state density is not a by-product of a "standard" vertical TD-DFT calculation, but requires the computation of the $\mathrm{Z}$-vector available through the computation of TD-DFT forces, that is the computational cost of an excited-state density calculation is similar to the one of a single TD-DFT gradient [22].

Vibrationally resolved spectra within the harmonic approximation were computed using the FCclasses program (FC) [30,45-47]. The reported spectra have been simulated using a convoluting Gaussian function presenting a half width at half maximum of $0.08 \mathrm{eV}$. A maximal number of 25 overtones for each mode and 20 combination bands on each pair of modes were included. The maximum number of integrals to be computed for each class was set to $10^{8}$, allowing to reach a FC factor larger than 0.95 for the treated cases.

\section{Results and discussion}

\subsection{Basis set effects}

In Table 1, we report the variations of selected quantities determined for $\mathbf{1}$ as a function of the selected atomic basis set, all calculations (geometry and transition energies) being performed with the same basis set. One clearly notices that there are only negligible variations between $6-31+\mathrm{G}(\mathrm{d})$ and $6-311+\mathrm{G}(2 \mathrm{~d}, \mathrm{p})$, whereas the omission of diffuse orbitals implies a $0.05-0.10 \mathrm{eV}$ increase of the transition energies, but essentially no variations of the difference of ZPVE between the two states. These conclusions are in the line of previous works [33, $42,48]$, and indicate that boricos are not particularly sensitive to basis set effects.

By correcting the $6-31 \mathrm{G}(\mathrm{d})$ results by $6-311+\mathrm{G}(2 \mathrm{~d}, \mathrm{p})$ vertical calculations [35], one reaches convergence. Indeed, the best estimates of the absorption-fluorescence crossing point ( $E^{\mathrm{AFCP}}$ ) of $\mathbf{1}$ at the (SS,neq)-PCM level obtained in that way are $2.97,2.96$ and $2.97 \mathrm{eV}$, when
Table 1 Basis set effects on the vertical absorption $\left(E^{\text {vert }-\mathrm{a}}\right)$, vertical emission $\left(E^{\text {vert }-\mathrm{f}}\right)$ and adiabatic $E^{\text {adia }}$ energies, as well as on the difference of zero point energies between the excited and ground states $\left(\Delta E^{\mathrm{ZPVE}}\right)$ of 1 . The selected PCM models are indicated. All values are in $\mathrm{eV}$.

\begin{tabular}{lllll}
\hline Basis set & $\begin{array}{l}E^{\text {vert-a }} \\
\text { cLR,neq }\end{array}$ & $\begin{array}{l}E^{\text {vert-f }} \\
\text { cLR,neq }\end{array}$ & $\begin{array}{l}E^{\text {adia }} \\
\text { LR,eq }\end{array}$ & $\begin{array}{l}\Delta E^{\text {ZPVE }} \\
\text { LR,eq }\end{array}$ \\
\hline 6-31G(d) & 3.29 & 2.88 & 2.90 & -0.08 \\
6-31+G(d) & 3.22 & 2.84 & 2.85 & -0.09 \\
$6-311+\mathrm{G}(2 \mathrm{~d}, \mathrm{p})$ & 3.21 & 2.83 & 2.84 & -0.08 \\
\hline
\end{tabular}

using 6-31G(d), 6-31+G(d) and 6-311+G(2d,p) structures, respectively, confirming that the smallest basis set is completely sufficient to describe the excited-state potential energy surface, at least around its minimum. In Figure 2, it is also obvious that the band topologies computed for both absorption and emission of $\mathbf{1}$ are converged with $6-31 \mathrm{G}(\mathrm{d})$, the variations obtained with $6-311+\mathrm{G}(2 \mathrm{~d}, \mathrm{p})$ being completely trifling.

In short this Section confirms that the AFCP values reported below are free of significant basis set effects.

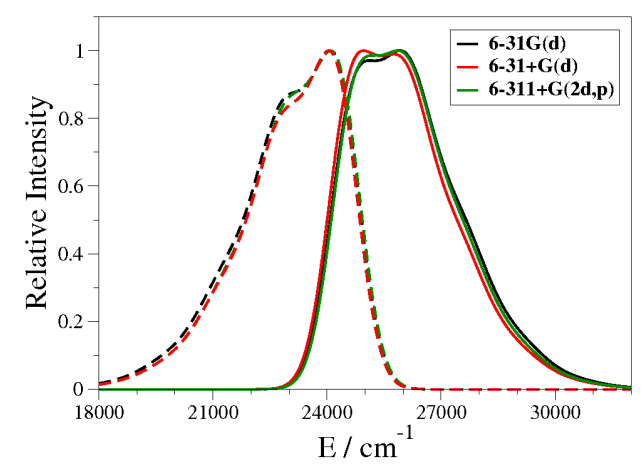

Fig. 2 Computed absorption (full lines) and emission (dashed lines) band shapes for $\mathbf{1}$ using three increasingly large atomic basis sets.

\subsection{Nature of the excited-states and 0-0 energies}

The density difference plot corresponding to the first excited-state of $\mathbf{2 a}$ is displayed at the top of Figure 3. As can be seen, the transition has a rather quadrupolar nature with charge-transfer (CT) from both ends (the diethylamino and benzimidazole moieties, mostly in blue) towards the two central six-membered rings (mostly in red). Clearly, the methyl group attached at the bottom of the phenyl ring does not play a major role, which explains why the visible spectra of $\mathbf{2 a}, \mathbf{2} \mathbf{b}$ and $\mathbf{2 c}$ are very similar experimentally (see below) [10]. In these three dyes, transitions towards the second and third singlet excited-states are less probable (smaller 
oscillator strength) than for the first excited-state, and these second and third state lie more than $1 \mathrm{eV}$ above the first, so that there is no need to discuss them further.

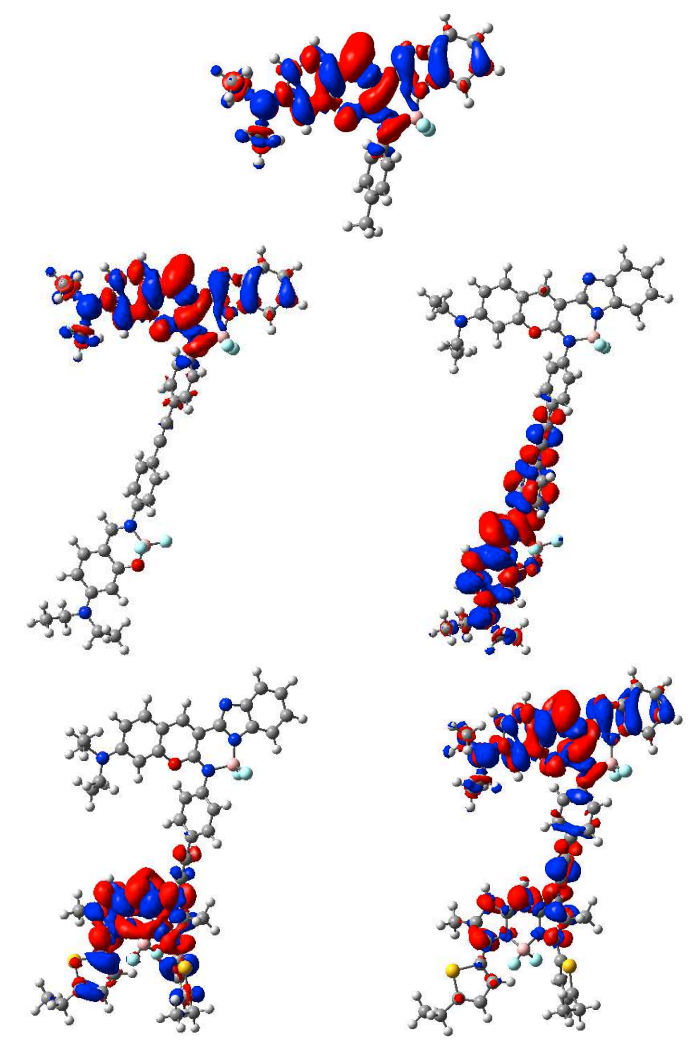

Fig. 3 Computed density difference plots for, from top to bottom, 2a, 3 and $\mathbf{4}$. For the two latter, the first (left) and second (right) excited-states are considered. All figures use a contour threshold of $5 \times 10^{-4}$ a.u.; the red (blue) regions indicating increase (decrease) of the electronic density upon electronic transition.

For the borico-boranil dyad, 3, the experiment indicates a nearly perfectly additive spectra, the borico absorbing at longer wavelength. This is completely consistent with the representation of Figure 3, where the first excited-state is clearly characteristic of the borico, whereas the second is localized on the boranil, without any significant overlap between the two. For the borico-BODIPY dyad, 4, the first state is typical of the BODIPY fluorophore and this is again consistent with experiment that finds the hallmark sharp and intense absorption and emission bands of BODIPY for this structure. The second excited-state is mainly centered on the borico but slightly extends on the BODIPY that acts as an acceptor (mostly in red). This slight overlap between the two first excited-states implies a very efficient energy transfer between the two subunits, that is also observed experimentally [10].

Table 2 provides a comparison between theoretical and experimental data for the five synthetically available boricos. Two conclusions emerge: on the one hand, TD-DFT provides too large transition energies in all cases, and, on the other hand, the global trends are correctly reproduced by theory. For the first aspect, the errors on the AFCP energies, the parameter affording the most direct comparison between measurements and simulations, are of the order of $0.4 \mathrm{eV}$ (but for the BODIPY, a case extensively studied elsewhere [38]), which is clearly above the usual TD-DFT error $(0.2-0.3 \mathrm{eV})$ $[33,35,36]$. Briefly, we can attribute this fact, not only to the choice of an exchange-correlation functional that contains a large amount of exact exchange, but also to the partial cyanine nature of the excited-state of fluoroborate complexes. Indeed, it is well-known that linear-response TD-DFT tend to overshoot the transition energies of cyanine structures (by ca. 0.20-0.60 $\mathrm{eV}$ ) compared to accurate reference data $[27,28,49,50]$. Whilst the use of the most modern exchange-correlation functionals, such as M06-2X [28] or optimally-tuned range separated hybrids [50], alleviate TD-DFT's error for cyanines making the obtained absolute deviation more in the line of other states, all tested TD-DFT protocols tend, to the best of our knowledge, to provide too large transition energies for this family of dyes.

In terms of chemical ranking, TD-DFT is much more satisfactory. Indeed, the small successive bathochromic shifts observed for the absorption/emission wavelengths in the $\mathbf{2} \mathbf{a} \rightarrow \mathbf{2} \mathbf{c} \rightarrow \mathbf{3}$ series are reproduced, e.g., +4 $(+6) \mathrm{nm}$ and $+5(+5) \mathrm{nm}$ variations of the fluorescence wavelengths with TD-DFT (experimentally). The same holds for the AFCP energies of the five compounds. The selected approach also restores the facts that the boranil absorption is much more intense than its borico counterpart, and the relatively small Stokes shifts obtained for BODIPY compared to boricos, though, for the latter case, the absolute values are significantly exaggerated in the vertical approximation.

\subsection{Band shapes}

To compute the band shapes, we have used the model molecule $\mathbf{1}$, in order to ensure the full convergence of the FC calculations. However, we have seen above that the absorption was centered on the borico core, and experimentally, the band topologies of $\mathbf{2 a}, \mathbf{2} \mathbf{b}$ and $\mathbf{2} \mathbf{c}$ are completely similar [10], hinting that this simplification is not an issue. The results of the stick and convoluted spectra are displayed in Figure 4, and the experimental shape is correctly reproduced with a double max- 
Table 2 Comparisons between experimental and theoretical 6-31G(d) values for characteristic data. Wavelengths are in $\mathrm{nm}$, the Stokes shifts $\left(\Delta_{\mathrm{SS}}^{\text {vert }}\right)$ are in $\mathrm{cm}^{-1}$ and energies are in $\mathrm{eV}$. For the absorption the values between brackets are the oscillator strength and $(\epsilon)$ for theory and experimental data, respectively. The experimental values are from Ref. [10]. All theoretical values determined at the cLR level (but $\Delta E^{\mathrm{ZPVE}}$ ) in the non-equilibrium limit but for $E^{\text {adia }}$ and $\Delta E^{\mathrm{ZPVE}}$.

\begin{tabular}{|c|c|c|c|c|c|c|}
\hline & \multicolumn{2}{|c|}{$\lambda^{\text {vert }-\mathrm{a}}$} & \multicolumn{2}{|c|}{$\lambda^{\text {vert }-\mathrm{f}}$} & \multicolumn{2}{|c|}{$\Delta_{\mathrm{SS}}^{\text {vert }}$} \\
\hline & Th. & Exp. & Th. & Exp. & Th. & Exp. \\
\hline $2 \mathbf{a}$ & 387 (1.08) & $492(41000)$ & 449 & 539 & 3547 & 1800 \\
\hline $2 b$ & 387 (1.09) & $492(45000)$ & 450 & 539 & 3631 & 1800 \\
\hline $2 c$ & 391 (1.05) & $496(47000)$ & 453 & 545 & 3504 & 1800 \\
\hline \multirow[t]{2}{*}{3} & $394(0.97)$ & $496(46000)$ & 459 & 550 & 3570 & 1900 \\
\hline & $352(2.42)$ & $420(85000)$ & & & & \\
\hline \multirow[t]{4}{*}{4} & $533(0.94)$ & $639(48000)$ & 586 & 691 & 1689 & 1300 \\
\hline & $403(0.82)^{a}$ & $497(60000)$ & & & & \\
\hline & $E^{\text {adia }}$ & $\Delta E^{\mathrm{ZPVE}}$ & \multicolumn{2}{|c|}{$E^{\mathrm{AFCP} b}$} & & \\
\hline & Th. & Th. & Th. & Exp. & & \\
\hline $\mathbf{2 a}$ & 2.98 & -0.07 & 2.87 & 2.41 & & \\
\hline $2 b$ & 2.97 & -0.07 & 2.87 & 2.41 & & \\
\hline 2c & 2.94 & -0.08 & 2.84 & 2.39 & & \\
\hline 3 & 2.90 & -0.07 & 2.80 & 2.38 & & \\
\hline 4 & 2.22 & -0.05 & 2.12 & 2.02 & & \\
\hline
\end{tabular}

${ }^{a}$ The third state is very close, $388 \mathrm{~nm}(0.75)$, the experimental band being probably the convolution of these two states ${ }^{b}$ Including basis set corrections with the $6-311+\mathrm{G}(2 \mathrm{~d}, \mathrm{p})$

ima for both absorption and emission, and an emission slightly departing from perfect mirror-image (see Figures $1 \mathrm{a}$ and $1 \mathrm{~b}$ of Ref. [10]).

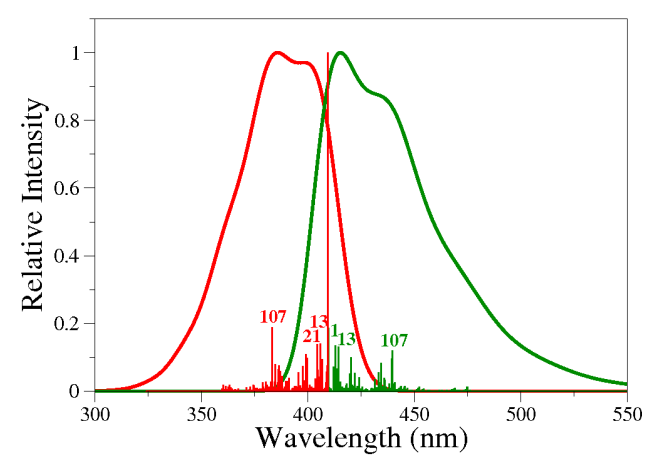

Fig. 4 Stick and convoluted spectra for absorption (red) and emission (green) spectra of $\mathbf{1}$.

As can be seen the vibrational mode that contributes the most significantly to the second maxima is mode $\mathrm{n}^{\circ} 107$ for both absorption and emission, a mode that has a circular frequency of 1667 and $1691 \mathrm{~cm}^{-1}$ in the excited and ground-state, respectively. Movies of these vibrational normal modes are given in the Supporting Information (SI), and they correspond to CC elongation centered on the central six-membered cycles containing the oxygen atom and the fluoroborate group. There- fore, this vibration and, consequently, the band shape are a typical signature of borico and cannot be obtained considering only an isolated coumarin or a BODIPY derivative.

\subsection{Design of new boricos}

In this Section, we have started with the borico core and evaluated the impact of push $\left(\mathrm{NMe}_{2}\right)$ and pull $\left(\mathrm{NO}_{2}\right)$ groups placed at the borders of the molecule (see Fig1800 ure 5). We aim not only to better understand the na00 ture of their excited-states but also to provide strategies to effectively tune the spectral signatures, the boricos proposed up to now absorbing at relatively short wavelength $(<500 \mathrm{~nm}$ experimentally [10]). Of course, our simulated values are shifted by ca. $0.4 \mathrm{eV}$ compared to experiment, but we expect the trends to be correctly reproduced (see Section 3.2).

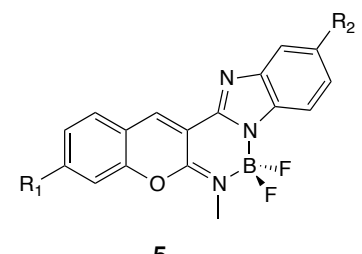

5

Fig. 5 Representation of the substitution positions selected for the push-pull design.

Our results are collected in Table 3, whereas Figure 6 provides density different plots for four selected derivatives. Starting with the hydrogen-capped dye, $5 \mathbf{a}$, one obtains bathochromic shifts for all substitutions but the addition of an accepting group on the benzimidazole 5b. This can be explained from Figure 6. Indeed, in $\mathbf{5 a}$, the benzimidazole clearly acts as an electron donor, and the coumarin as the acceptor. Therefore 5b implies a weak charge-transfer, as the benzimidazole moiety donating power strongly falls. For $\mathbf{5 e}(=\mathbf{1})$, one reaches a double CT from the side to the center (see Section 3.2). Clearly increasing the strength of the benzimidazole $\mathbf{5 c}$ is the most efficient strategy to move the transition energies, this simple substitution leading to a bathochromic shift of ca. $90 \mathrm{~nm}$. When performing a double substitution, it is therefore logically $\mathbf{5 i}$ that is the most effective, with an additional redshift of ca. $100 \mathrm{~nm}$, creating a strongly dipolar CT transition as seen in Figure 6. This success is however at the price of a smaller oscillator strength. For 5i, we compute a CT distance of $5.6 \AA$ and an amount of charge transferred close to $1(0.98 e)$, within Le Bahers model [51], that are very large values, amongst the best for 
push-pull systems [52], the distance being in fact at least ca. 1.0 $\AA$ larger than the one computed for other molecules in Table 3. On the contrary, the reverse strategy, 5g, provides significantly larger transition energies with an excited-state mostly localized on the coumarin side. The double-donor compound (5f) presents a CT of quadrupolar nature (see Figure 6), with transition energies very similar to the one of $\mathbf{5 e}$.

Table 3 Predicted PCM(cLR,neq)-TD-M06-2X/6-31G(d) transition results for boricos $\mathbf{5}$ (see Figure 5). Wavelengths in $\mathrm{nm}, \mathrm{AFCP}$ energies in $\mathrm{eV}$.

\begin{tabular}{llllll}
\hline & $\mathrm{R}_{1}$ & $\mathrm{R}_{2}$ & $\lambda^{\text {vert-a }}$ & $\lambda^{\text {vert-f }}$ & $E^{\mathrm{AFCP} a}$ \\
\hline $\mathbf{5 a}$ & $\mathrm{H}$ & $\mathrm{H}$ & $347(0.80)$ & 448 & 3.03 \\
$\mathbf{5 b}$ & $\mathrm{H}$ & $\mathrm{NO}_{2}$ & $330(0.93)$ & 399 & 3.31 \\
$\mathbf{5 c}$ & $\mathrm{H}$ & $\mathrm{NMe}_{2}$ & $434(0.46)$ & 847 & 1.97 \\
$\mathbf{5 d}$ & $\mathrm{NO}_{2}$ & $\mathrm{H}$ & $389(0.87)$ & 526 & 2.67 \\
$\mathbf{5 e}$ & $\mathrm{NMe}_{2}$ & $\mathrm{H}$ & $374(1.16)$ & 431 & 2.97 \\
$\mathbf{5 f}$ & $\mathrm{NMe}_{2}$ & $\mathrm{NMe}_{2}$ & $395(1.13)$ & 539 & 2.43 \\
$\mathbf{5 g}$ & $\mathrm{NMe}_{2}$ & $\mathrm{NO}_{2}$ & $374(1.26)$ & 416 & 3.01 \\
$\mathbf{5 h}$ & $\mathrm{NO}_{2}$ & $\mathrm{NO}_{2}$ & $358(1.01)$ & 472 & 2.91 \\
$\mathbf{5 i}$ & $\mathrm{NO}_{2}$ & $\mathrm{NMe}_{2}$ & $533(0.44)$ & 1135 & 1.63 \\
\hline
\end{tabular}

${ }^{a}$ Including basis set corrections with the $6-311+\mathrm{G}(2 \mathrm{~d}, \mathrm{p})$
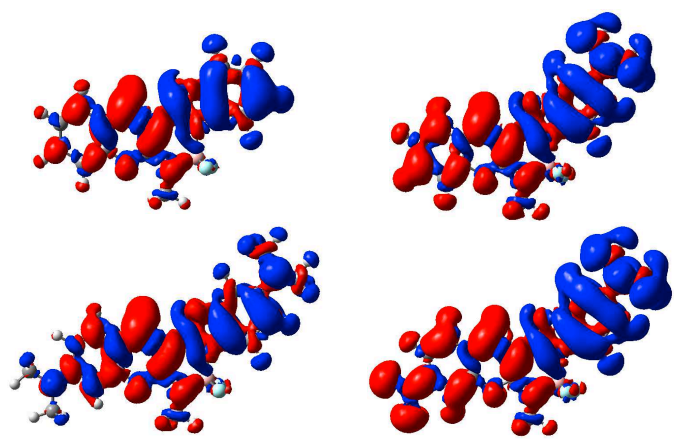

Fig. 6 Computed density difference plots for $\mathbf{5 a}$ (top left), 5c (top right), 5f (bottom left) and 5i (bottom right). See caption of Figure 3 for more details.

\subsection{Bochkov's dyes}

As stated in the Introduction, new coumarin-BODIPY structures, presenting a completely different architecture, appeared two months ago [12]. These molecules are displayed in Figure 7, and Table 3 reports key data computed with exactly the same computational protocol as the one used above. In this series, the second singlet excited-state is at least $0.8 \mathrm{eV}$ above the first and present a smaller oscillator strength, so that we report and discuss only the data obtained for the first excited-state. Though, as expected, the theoretical transition energies are systematically too large, the trends are nicely reproduced in the $6 \mathbf{a} \rightarrow \mathbf{6 b} \rightarrow \mathbf{6 c} \rightarrow$ 6d series: i) the successive shifts of the AFCP energies are $-0.08(-0.08),-0.27(-0.35)$ and $-0.01(-0.01) \mathrm{eV}$ with TD-DFT (experimentally); ii) the computed evolution of the absorption oscillator strengths perfectly follows the experimental intensities. As for the other series, the order of magnitude of the Stokes shifts is reproduced, but its significant increase for the diethyl amino substituted dyes is not.

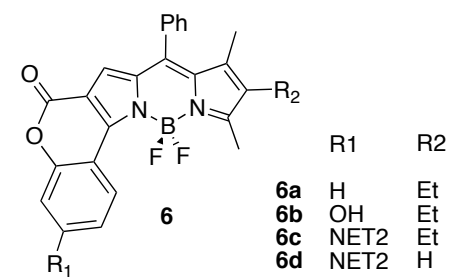

Fig. 7 Representation of four fused coumarin-BODIPY dyes under study.

Table 4 Comparisons between experimental and theoretical values for the dyes of Figure 7 in DCM. See caption of Table 2 for more details.

\begin{tabular}{|c|c|c|c|c|c|c|}
\hline & \multicolumn{2}{|c|}{$\lambda^{\text {vert-a }}$} & \multicolumn{2}{|c|}{$\lambda^{\text {vert }-\mathrm{f}}$} & \multicolumn{2}{|c|}{$\Delta_{\mathrm{SS}}^{\text {vert }}$} \\
\hline & Th. & Exp. & Th. & Exp. & Th. & Exp. \\
\hline $6 \mathbf{a}$ & $429(0.79)$ & $516(36000)$ & 466 & 546 & 1841 & 1065 \\
\hline $6 \mathrm{~b}$ & $444(0.82)$ & $530(46200)$ & 482 & 567 & 1816 & 1231 \\
\hline $6 c$ & $492(0.94)$ & $602(59400)$ & 544 & 697 & 1944 & 2264 \\
\hline \multirow[t]{3}{*}{$6 d$} & $491(0.93)$ & $606(59100)$ & 541 & 706 & 1876 & 2337 \\
\hline & $E^{\text {adia }}$ & $\Delta E^{\mathrm{ZPVE}}$ & \multicolumn{2}{|c|}{$E^{\mathrm{AFCP} a}$} & & \\
\hline & Th. & Th. & Th. & Exp. & & \\
\hline $6 \mathbf{a}$ & 2.54 & -0.07 & 2.69 & 2.34 & & \\
\hline $6 \mathrm{~b}$ & 2.45 & -0.07 & 2.61 & 2.26 & & \\
\hline $6 c$ & 2.23 & -0.04 & 2.34 & 1.91 & & \\
\hline $6 d$ & 2.23 & -0.04 & 2.33 & 1.90 & & \\
\hline
\end{tabular}

${ }^{a}$ Including basis set corrections with the $6-311+\mathrm{G}(2 \mathrm{~d}, \mathrm{p})$

The vibronic spectra for a model molecule presenting the skeleton of $\mathbf{6}$ are shown in Figure 8, whereas Figure 9 gives the density difference plots obtained for 6a and 6c. On the former graph, one notices the presence of a shoulder, consistently with experiment [12], but its height is slightly underestimated, a result typical of BODIPY structures [38]. The changes compared to Figure 4 are nevertheless obvious and in the line of the measurements. The vibrations significantly contributing to the shoulder of Figure 8 are CC stretching located on the coumarin ring and on the pyrrole bonded to it. In Figure 9 , one notices a significant CT from the 
coumarin to the BODIPY core, this CT being enhanced by the $\mathrm{NEt}_{2}$ group.

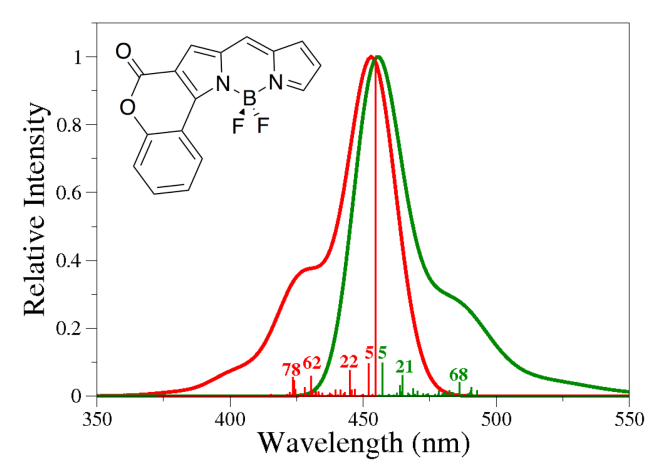

Fig. 8 Stick and convoluted spectra for absorption (red) and emission (green) spectra of a model 6 .

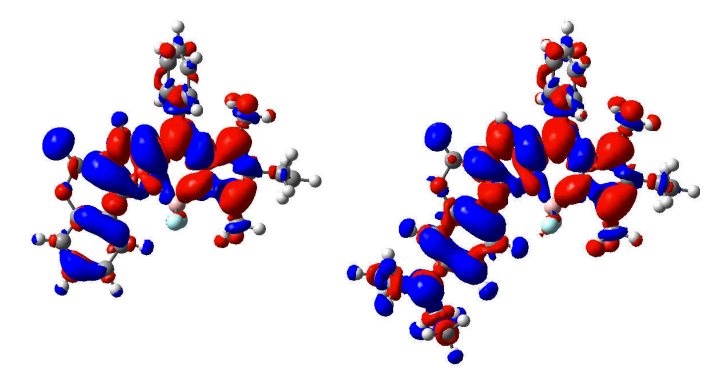

Fig. 9 Density difference plots for 6a (left) and 6c (right). See caption of Figure 3 for more details.

\section{Conclusions}

We have used TD-DFT to rationalize the spectral properties of new promising fluorophores, boricos, that combine, in a single conjugated core, a coumarin and a fluoroborate complex, both subunits being strongly emissive. The selected TD-DFT approach is able to reproduce several experimental features: auxochromic shifts, relative ordering of the bands in large dyads, as well as characteristic band shapes of boricos. There is however a systematic overestimation of the transition energies, an error that cannot be attributed to basis set effects nor, most probably, solvation (a refined PCM model was selected and the selected solvent is aprotic), but more likely to the partial cyanine nature of the considered excited-states. A series of new molecules have been proposed to the synthesis, and it indicates that adding an electron acceptor on the coumarin side and a strong pulling group on the benzimidazole moiety is the most efficient strategy to obtain a large redshift. In fact, the dialkylamino group presents experimentally on the coumarin side is predicted to have a rather minor impact $(\sim 30 \mathrm{~nm})$ on the absorption wavelength of boricos compared to a simple hydrogen atom. For chromophores presenting a fused coumarin-BODIPY core, theory allowed to pinpoint the strong CT character for the experimental band. It is our hope that the present contribution will stimulate more experimental work for these emerging classes of compounds.

Acknowledgements A.C.E thank the European Research Council (ERC, Marches - 278845) and the Région des Pays de la Loire for his postdoctoral grants. D.J. acknowledges the European Research Council (ERC) and the Région des Pays de la Loire for financial support in the framework of a Starting Grant (Marches - 278845) and a recrutement sur poste stratégique, respectively. This research used resources of 1) the GENCI-CINES/IDRIS (Grants c2013085117 and x2013080649), 2) CCIPL (Centre de Calcul Intensif des Pays de Loire) and 3) a local Troy cluster.

\section{References}

1. A. Loudet, K. Burgess, Chem. Rev. 107, 4891 (2007)

2. G. Ulrich, R. Ziessel, A. Harriman, Angew. Chem. Int. Ed. 47, 1184 (2008)

3. A. Coskun, M. Yilmaz, E.U. Akkaya, Org. Lett. 9, 607 (2007)

4. J. Murtagh, D.O. Frimannsson, D.F. O'Shea, Org. Lett. 11, 5386 (2009)

5. Q. Zheng, G. Xu, P. Prasad, Chem. Eur. J. 14(19), 5812 (2008)

6. J. Killoran, L. Allen, J.F. Gallagher, W.M. Gallagher, D.F. O'Shea, Chem. Commun. pp. 1862-1863 (2002)

7. J.V. Frangioni, Curr. Opin. Chem. Biol. 7, 626 (2003)

8. D. Jacquemin, E.A. Perpète, G. Scalmani, M.J. Frisch, X. Assfeld, I. Ciofini, C. Adamo, J. Chem. Phys. 125, $164324(2006)$

9. S. Banerjee, E.B. Veale, C.M. Phelan, S.A. Murphy, G.M. Tocci, L.J. Gillespie, D.O. Frimannsson, J.M. Kelly, T. Gunnlaugsson, Chem. Soc. Rev. 42, 1601 (2013)

10. D. Frath, A. Poirel, G. Ulrich, A. De Nicola, R. Ziessel, Chem. Commun. 49, 4908 (2013)

11. X. Cao, W. Lin, Q. Yu, J. Wang, Org. Lett. 13(22), 6098 (2011)

12. A.Y. Bochkov, I.O. Akchurin, O.A. Dyachenko, V.F. Traven, Chem. Commun. pp. - (2013)

13. M.E. Casida, Time-Dependent Density-Functional Response Theory for Molecules (World Scientific, Singapore, 1995), Recent Advances in Density Functional Methods, vol. 1, pp. 155-192

14. K. Burke, J. Werschnik, E.K.U. Gross, J. Chem. Phys. 123, 062206 (2005)

15. M.A.L. Marques, F.M.S. Nogueira, E.K.U. Gross, A. Rubio (eds.), Fundamentals of Time-Dependent Density Functional Theory, Lecture Notes in Physics, vol. 837 (Springer-Verlag, Heidelberg, 2012)

16. C. Adamo, D. Jacquemin, Chem. Soc. Rev. 42, 845 (2013)

17. L. Ordronneau, V. Aubert, V. Guerchais, A. Boucekkine, H. Le Bozec, A. Singh, I. Ledoux, D. Jacquemin, Chem. Eur. J. 19, 5845 (2013) 
18. S. Grimme, J. Chem. Phys. 138, 244104 (2013)

19. J. Tomasi, B. Mennucci, R. Cammi, Chem. Rev. 105, $2999(2005)$

20. D. Jacquemin, B. Mennucci, C. Adamo, Phys. Chem. Chem. Phys. 13, 16987 (2011)

21. C. van Caillie, R.D. Amos, Chem. Phys. Lett. 308, 249 (1999)

22. F. Furche, R. Ahlrichs, J. Chem. Phys. 117, 7433 (2002)

23. G. Scalmani, M.J. Frisch, B. Mennucci, J. Tomasi, R. Cammi, V. Barone, J. Chem. Phys. 124, 094107 (2006)

24. J. Liu, W.Z. Liang, J. Chem. Phys. 135(1), 014113 (2011)

25. J. Liu, W.Z. Liang, J. Chem. Phys. 135(18), 184111 (2011)

26. S. Grimme, F. Neese, J. Chem. Phys. 127, 154116 (2007)

27. J. Fabian, Dyes Pigm. 84, 36 (2010)

28. D. Jacquemin, Y. Zhao, R. Valero, C. Adamo, I. Ciofini, D.G. Truhlar, J. Chem. Theory Comput. 8, 1255 (2012)

29. M. Dierksen, S. Grimme, J. Phys. Chem. A 108, 10225 (2004)

30. F. Santoro, R. Improta, A. Lami, J. Bloino, V. Barone, J. Chem. Phys. 126, 084509 (2007)

31. A. Charaf-Eddin, A. Planchat, B. Mennucci, C. Adamo, D. Jacquemin, J. Chem. Theory Comput. 9, 2749 (2013)

32. B. Pritchard, J. Autschbach, ChemPhysChem 11(11), 2409 (2010)

33. L. Goerigk, S. Grimme, J. Chem. Phys. 132, 184103 (2010)

34. R. Send, M. Kühn, F. Furche, J. Chem. Theory Comput. 7(8), 2376 (2011)

35. D. Jacquemin, A. Planchat, C. Adamo, B. Mennucci, J. Chem. Theory Comput. 8, 2359 (2012)

36. N.O.C. Winter, N.K. Graf, S. Leutwyler, C. Hattig, Phys. Chem. Chem. Phys. 15, 6623 (2013)

37. M.J. Frisch, G.W. Trucks, H.B. Schlegel, G.E. Scuseria, M.A. Robb, J.R. Cheeseman, G. Scalmani, V. Barone, B. Mennucci, G.A. Petersson, H. Nakatsuji, M. Caricato, X. Li, H.P. Hratchian, A.F. Izmaylov, J. Bloino, G. Zheng, J.L. Sonnenberg, M. Hada, M. Ehara, K. Toyota, R. Fukuda, J. Hasegawa, M. Ishida, T. Nakajima, Y. Honda, O. Kitao, H. Nakai, T. Vreven, J.A. Montgomery, Jr., J.E. Peralta, F. Ogliaro, M. Bearpark, J.J. Heyd, E. Brothers, K.N. Kudin, V.N. Staroverov, R. Kobayashi, J. Normand, K. Raghavachari, A. Rendell, J.C. Burant, S.S. Iyengar, J. Tomasi, M. Cossi, N. Rega, J.M. Millam, M. Klene, J.E. Knox, J.B. Cross, V. Bakken, C. Adamo, J. Jaramillo, R. Gomperts, R.E. Stratmann, O. Yazyev, A.J. Austin, R. Cammi, C. Pomelli, J.W. Ochterski, R.L. Martin, K. Morokuma, V.G. Zakrzewski, G.A. Voth, P. Salvador, J.J. Dannenberg, S. Dapprich, A.D. Daniels, O. Farkas, J.B. Foresman, J.V. Ortiz, J. Cioslowski, D.J. Fox. Gaussian 09 Revision D.01 (2009). Gaussian Inc. Wallingford CT

38. S. Chibani, B. Le Guennic, A. Charaf-Eddin, A.D. Laurent, D. Jacquemin, Chem. Sci. 4, 1950 (2013)

39. S. Chibani, A. Charaf-Eddin, B. Le Guennic, D. Jacquemin, J. Chem. Theory Comput. 9, 3127 (2013)

40. Y. Zhao, D.G. Truhlar, Theor. Chem. Acc. 120, 215 (2008)

41. M. Caricato, B. Mennucci, J. Tomasi, F. Ingrosso, R. Cammi, S. Corni, G. Scalmani, J. Chem. Phys. 124, $124520(2006)$

42. S. Chibani, B. Le Guennic, A. Charaf-Eddin, O. Maury, C. Andraud, D. Jacquemin, J. Chem. Theory Comput. 8, 3303 (2012)
43. W.J. Stevens, H. Basch, M. Krauss, J. Chem. Phys. 81, 6026 (1984)

44. W.J. Stevens, M. Krauss, H. Basch, P.G. Jasien, Can. J. Chem. 70, 612 (1992)

45. F. Santoro, R. Improta, A. Lami, J. Bloino, V. Barone, J. Chem. Phys. 126, 184102 (2007)

46. F. Santoro, A. Lami, R. Improta, J. Bloino, V. Barone, J. Chem. Phys. 128, 224311 (2008)

47. F.J. Avila Ferrer, J. Cerezo, E. Stendardo, R. Improta, F. Santoro, J. Chem. Theory Comput. 9, 2072 (2013)

48. D. Jacquemin, C. Adamo, Int. J. Quantum Chem. 112, 2135 (2012)

49. R. Send, O. Valsson, C. Filippi, J. Chem. Theory Comput. $7(2), 444(2011)$

50. B. Moore II, J. Autschbach, J. Chem. Theory Comput. 9, $4991(2013)$

51. T. Le Bahers, C. Adamo, I. Ciofini, J. Chem. Theory Comput. 7(8), 2498 (2011)

52. I. Ciofini, T. Le Bahers, C. Adamo, F. Odobel, D. Jacquemin, J. Phys. Chem. C 116, 11946 (2012), erratum: ibidem 14736-14736 